\title{
Investigating racial differences in clinical and pathological features of prostate cancer in South African men
}

\author{
M Dewar, L Kaestner, Q Zikhali, K Jehle, S Sinha, J Lazarus \\ Division of Urology, Groote Schuur Hospital and the University of Cape Town, Cape Town, South Africa
}

Corresponding author: Malcolm James Dewar(maldewar@gmail.com)

\begin{abstract}
Introduction: Men with West African ancestry living in Europe and North America are at higher risk of being diagnosed with prostate cancer, are diagnosed at a younger age, and have more severe disease characteristics. Published reports present a conflicting picture of the disease in sub-Saharan Africa. We aimed to study the clinical and pathological features of men undergoing prostate biopsy from different racial backgrounds in South Africa in an attempt to characterise the disease locally. Our hypothesis was that black African men presenting to our service had more severe disease characteristics than other patients.

Methods: All patients who underwent a prostate biopsy at Groote Schuur Hospital, Cape Town from July 2008 to July 2014 were studied. For each patient, data were collected on age, self-assigned race, presenting symptoms, prostate-specific antigen (PSA) level, prostate volume, and histological diagnosis.

Results: A total of 1016 patients were studied. 162 (15.9\%) were black and $854(84.1 \%)$ were coloured (mixed ancestry), white, or Asian. Black patients were compared as a group to the coloured, white and Asian patients. The black patients in the series had higher PSA values (mean 167.8 vs 47.7, median 16.4 vs 10.9 , p $<0.001$ ), were more likely to be diagnosed with cancer $(57.4 \%$ vs $44.5 \%, \mathrm{p}=0.003)$, were more likely to present with locally advanced cancer $(\mathrm{T} 3 / 416.1 \%$ vs $8.9 \%$, $\mathrm{p}=0.028$ ), and were more likely to have high grade disease (Gleason $\geq 845.2 \%$ vs 30.5\%, $\mathrm{p}=0.011$ ). There was no difference in age, presenting symptoms, or prostate volume.

Conclusion: The black men diagnosed with prostate cancer at Groote Schuur Hospital had significantly worse clinical and pathological characteristics than the non-black men. Interpreting these differences as representative of a more common or aggressive disease among black men is not possible due to study limitations.
\end{abstract}

\section{Introduction}

Prostate Cancer (PCa) is one of the most common cancers diagnosed in men. ${ }^{1}$ In South Africa, prostate cancer is the most frequently diagnosed cancer in men, and is second only to lung cancer in terms of mortality. ${ }^{2}$

African American men have been shown to have a higher incidence of $\mathrm{PCa}$, higher stage at presentation, higher histological grade, a greater propensity to relapse after treatment, and a higher disease-specific mortality. ${ }^{3,4}$ Men with West-African ancestry living elsewhere, such as South America, the United Kingdom and the Caribbean, have similarly high rates of prostate cancer. ${ }^{5-7}$ Delayed diagnosis accounts in part for the higher stage at presentation and possibly some of the increased mortality. This delay in diagnosis has been attributed to healthcare access problems, reduced health-seeking behaviour, and often health-provider prejudice. ${ }^{8}$ Studies attempting to correct for these factors conclude that they do not account for all of the discrepancies seen and that some of the differences in disease characteristics must be due to differences in tumour biology between African American men and other American men. ${ }^{9}$ These are most likely the result of underlying genetic factors. ${ }^{4}$

By contrast, epidemiological studies on populations within Africa report far lower incidences of prostate cancer than those reported in populations of African descent in Europe and the Americas. ${ }^{10}$ There is some evidence, however, that these rates are misleadingly low due to underdiagnosis and underreporting of cases. ${ }^{11,12}$

Studies focused on South Africa have uniformly found $\mathrm{PCa}$ to be more common in white than black South Africans. ${ }^{2,13}$ Other studies have questioned this. ${ }^{14}$ These seemingly discordant findings suggest that prostate cancer in the South African black population might be underdiagnosed. Prostate cancer incidence within a population is heavily influenced by screening practices. Due to lower socio-economic status, the average black male in South Africa might be less likely to be 
screened for prostate cancer, and thus be less likely to have a prostate biopsy than one of his white, coloured or Asian counterparts. This is illustrated by the finding that the biopsyproven incidence in black men in South Africa is less than a quarter that of white South African men, but their mortality rate is more than double. ${ }^{2}$

We set out to analyse our biopsy database to characterise the clinical and pathological nature of PCa in black and nonblack men in the Western Cape. This data is vital to inform service provision planning. Our working hypothesis was that the black patients within our population might present with higher serum Prostate Specific Antigen (PSA) levels, larger prostates, more locally advanced tumours, and tumours of a higher histological grade. We also thought that black patients might be more likely to present with lower urinary tract symptoms, and less likely to have been biopsied as a result of PSA screening.

\section{Materials and Methods}

Approval for the study was obtained from the Human Research Ethics Committee of the University of Cape Town (REB\# 099/2015). A comprehensive literature review on the topic of prostate cancer and race was carried out using Medline and Google Scholar.

The study was performed within the Division of Urology at Groote Schuur Hospital in Cape Town, South Africa. Since 2008, the division has prospectively kept a database of all the patients undergoing prostate biopsy. All patients had been referred to the urology service via normal channels and had biopsies as part of routine clinical care. Indications for prostate biopsy included an elevated serum PSA level or an abnormal digital rectal examination (DRE). We routinely perform a 12 core transrectal ultrasound-guided biopsy under local anaesthetic.

A retrospective analysis of the database was performed, covering the patients biopsied between July 2008 and June 2014. Data points analysed were age, race, PSA value (in $\mathrm{ng} / \mathrm{ml}$ ), presenting symptoms, findings on DRE, and histopathology results (Gleason score). Men were excluded if PSA value, histology result or self-assigned race was not available. There was no attempt to impute other missing data points. Patients' self-assigned race, as recorded by hospital administration staff, was obtained from the electronic patient record database. The hospital record-keeping system uses the same race categories as those used in the South African census, namely Asian/Indian, Black, Coloured, and White. In keeping with our hypothesis that black patients might have worse clinical and pathological features, we compared the coloured, white and Asian patients as a group ("nonblack" patients) with the black patients. We examined the mode of presentation of the patients and classified them into four groups. Asymptomatic patients were those who presented requesting PSA screening without symptoms of prostate pathology, or those who were referred to us having had a screening PSA. Men presenting with symptoms were classified as having LUTS (obstructive or irritative lower urinary tract symptoms), symptoms of metastases (bone pain, loss of weight, paraplegia), or other (haematuria, haematospermia, etc.).

In the analysis, Gleason score was divided dichotomously in two different ways. Firstly, since Gleason 6 tumours are often considered insignificant, we compared Gleason 6 vs $\geq 7$. Secondly, since Gleason 8-10 tumours are often termed high grade, we compared those to Gleason $\leq 7$ tumours. Nominal variables (positive biopsy and Gleason score) were compared using Pearson's Chi-squared test, normally distributed data (age) were compared using the Welch two sample t-test, and non-parametric variables (PSA and prostate volume) were compared using the Wilcoxon rank sum test. Statistical significance was defined as a $\mathrm{p}$-value $\leq 0.05$.

\section{Results}

The database contained details on 1027 men. 11 patients were excluded from the analysis due to missing data on PSA value, race group, or histology results. Thus, a total of 1016 patients were included in the analysis. $162(25.9 \%)$ were black, $757(74.5 \%)$ were coloured, $89(8.8 \%)$ were white, and 8 $(0.8 \%)$ were Asian. The mean age of the cohort was 65.8 years, and was matched across race groups. Other missing data included 47 with no presenting symptom, 6 with no age, 87 with no DRE findings, and 24 with no prostate volume.

The rate of screening in this cohort was low, with only $21.5 \%$ of the patients being asymptomatic. The likelihood of a positive biopsy among the asymptomatic men was similar between the black and non-black patients (41.4\% vs $42.8 \%$, respectively). $77.2 \%$ presented with LUTS, $0.8 \%$ with symptoms of metastases, and $0.7 \%$ with other symptoms. There were no statistically significant differences between the race groups in terms of presenting symptoms $(p=0.731)$.

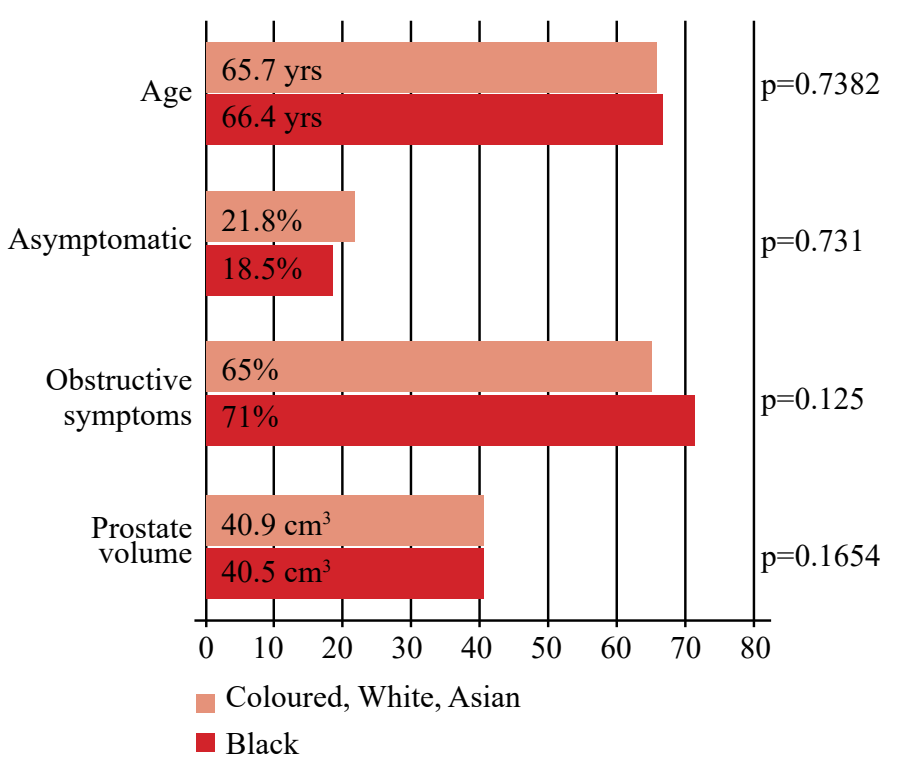

Figure 1: Mean age and symptom distribution; median prostate volume 
Patient-matched PSA values $\left(\log _{10}\right.$ scale $)$

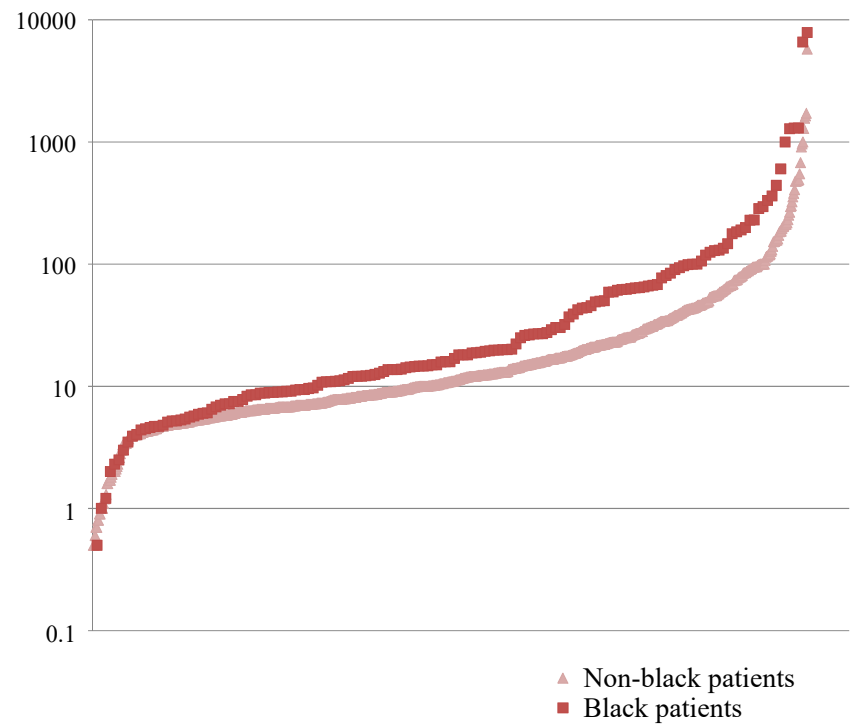

Figure 2: Scatter plot of all PSA values - black vs non-black $(p<0.001)$

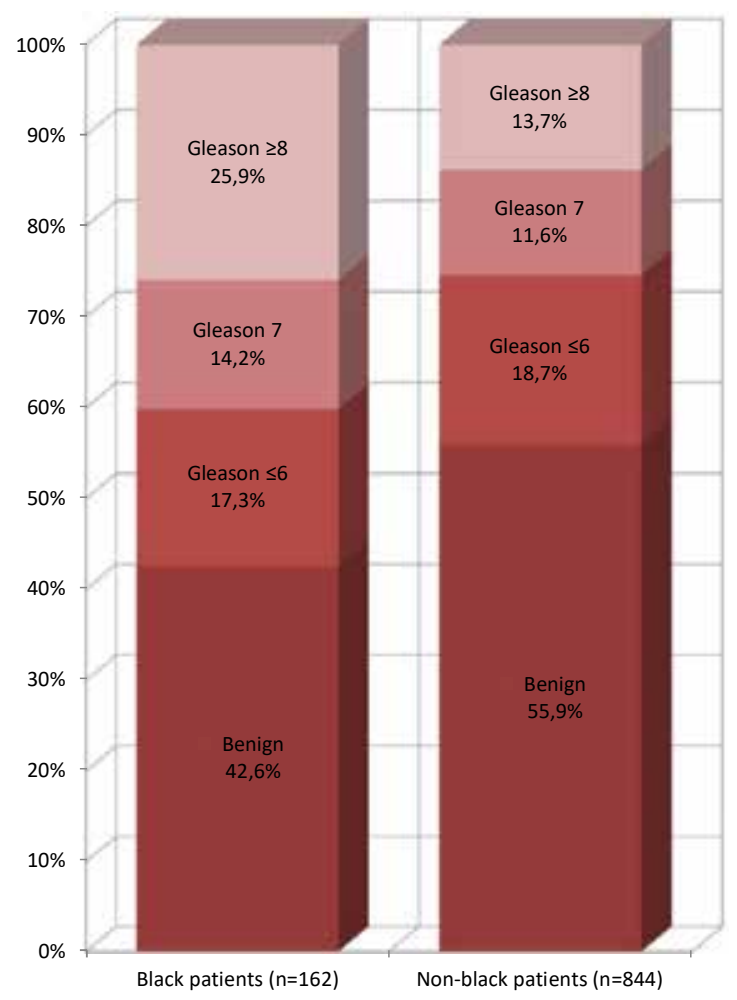

Figure 3: Histological diagnosis - black vs non-black

More black patients presented with obstructive urinary symptoms $(70.6 \%$ vs $64.3 \%)$, but this difference did not achieve statistical significance $(\mathrm{p}=0.127)$ (see Figure 1).

Black patients had significantly higher PSA values than the coloured, white and Asian patients $(\mathrm{p}<0.001)$. The mean PSA value among black patients was $167.8 \mathrm{ng} / \mathrm{ml}$, compared to $47.7 \mathrm{ng} / \mathrm{ml}$ in the non-black patients. The

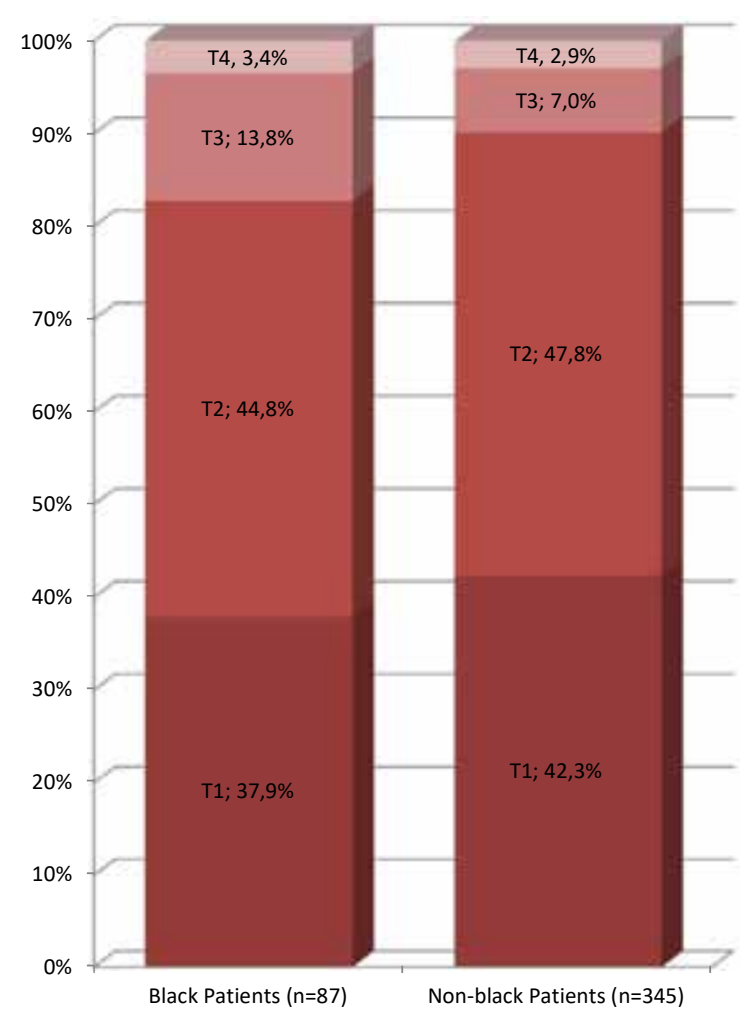

Figure 4: Digital rectal examination findings

mean values in both groups were somewhat skewed by a few very high outliers. Median PSA values were 16.4 and $10.9 \mathrm{ng} / \mathrm{ml}$, respectively (see Figure 2). Mean estimated prostate volume was higher in the black patients $(57.8 \mathrm{~g}$ vs $50.6 \mathrm{~g})$, but this was not statistically significant $(\mathrm{p}=0.165)$. There was also no statistically significant difference, with respect to race, in the PSA densities of the patients who had negative biopsies (mean PSA-d $0.433 \mathrm{ng} / \mathrm{ml} / \mathrm{ml}$ in black patients vs $0.376 \mathrm{ng} / \mathrm{ml} / \mathrm{ml}$ in non-black patients, $\mathrm{p}=0.3603)$. The overall positive biopsy rate was $46.6 \%$. Coloured, white and Asian patients had cancer diagnosed $44.5 \%$ of the time, while black patients had positive biopsies in $57.4 \%$. This translated into black patients having a $29 \%$ higher chance of being diagnosed with cancer, which was highly statistically significant $(\mathrm{p}=0.003)$. The mean ages of men with positive biopsies were similar between black and non-black patients (68.3 vs 67.2 years).

Higher grade tumours were noted in the black patients. They were significantly more likely to have tumours that were Gleason $\geq 7(74.4 \%$ vs $62.5 \%, \mathrm{p}=0.033)$ and Gleason $\geq 8$ $(51.1 \%$ vs $36.5 \%, p=0.011)$ (see Figure 3). Black patients were more likely to have extraprostatic disease clinically. Of the black patients diagnosed with cancer, 15/93 (16.1\%) had T3 or T4 disease on DRE, compared to $34 / 380$ (8.9\%) of nonblack patients $(\mathrm{p}=0.028)$ (see Figure 4$)$. 
Table 1 - Summary of results.

\begin{tabular}{|c|c|c|c|c|}
\hline & $\begin{array}{c}\text { Black } \\
\text { Patients } \\
\mathrm{n}=162 \\
(15.9 \%)\end{array}$ & $\begin{array}{c}\text { Non- } \\
\text { Black } \\
\text { Patients } \\
\mathbf{n}=\mathbf{8 5 4} \\
(\mathbf{8 4 . 1 \% )}\end{array}$ & $\begin{array}{c}\text { Total } \\
\mathrm{n}=1016\end{array}$ & p-value \\
\hline Asymptomatic & $\begin{array}{c}29 / 162 \\
(18.5 \%)\end{array}$ & $\begin{array}{l}177 / 854 \\
(21.8 \%)\end{array}$ & $\begin{array}{l}206 / 1016 \\
(21.2 \%)\end{array}$ & 0.731 \\
\hline $\begin{array}{l}\text { Obstructive } \\
\text { symptoms }\end{array}$ & $\begin{array}{l}115 / 162 \\
(71.0 \%)\end{array}$ & $\begin{array}{l}555 / 854 \\
(65.0 \%)\end{array}$ & $\begin{array}{c}670 / 1016 \\
(66.0 \%)\end{array}$ & 0.125 \\
\hline $\begin{array}{l}\text { PSA value in } \mathrm{ng} / \mathrm{ml} \\
\text { (mean / median) }\end{array}$ & $\begin{array}{c}166.8 / \\
16.4\end{array}$ & $\begin{array}{c}47.5 / \\
10.9\end{array}$ & $\begin{array}{c}66.6 / \\
12.0\end{array}$ & $<0.001$ \\
\hline $\begin{array}{l}\text { Prostate volume } \\
\text { (mean / median) }\end{array}$ & $\begin{array}{l}57.8 \mathrm{~g} / \\
40.5 \mathrm{~g}\end{array}$ & $\begin{array}{l}50.6 \mathrm{~g} / \\
40.9 \mathrm{~g}\end{array}$ & $\begin{array}{l}51.7 \mathrm{~g} / \\
41.0 \mathrm{~g}\end{array}$ & 0.165 \\
\hline Positive biopsy & $\begin{array}{c}93 \\
(57.4 \%)\end{array}$ & $\begin{array}{c}380 \\
(44.5 \%)\end{array}$ & $\begin{array}{c}473 \\
(46.6 \%)\end{array}$ & 0.003 \\
\hline Gleason $\geq 7$ & $\begin{array}{c}65 / 93 \\
(69.9 \%)\end{array}$ & $\begin{array}{l}214 / 380 \\
(56.3 \%)\end{array}$ & $\begin{array}{l}307 / 473 \\
(64.9 \%)\end{array}$ & 0.033 \\
\hline Gleason $\geq 8$ & $\begin{array}{c}42 / 93 \\
(45.2 \%)\end{array}$ & $\begin{array}{l}116 / 380 \\
(30.5 \%)\end{array}$ & $\begin{array}{l}158 / 473 \\
(33.4 \%)\end{array}$ & 0.012 \\
\hline DRE - T3/T4 & $\begin{array}{c}15 / 93 \\
(16.1 \%)\end{array}$ & $\begin{array}{l}34 / 380 \\
(8.9 \%)\end{array}$ & $\begin{array}{c}49 / 473 \\
(10.4 \%)\end{array}$ & 0.028 \\
\hline
\end{tabular}

\section{Discussion}

Worldwide variations in $\mathrm{PCa}$ incidence and aggressiveness exist in patients from different racial or ethnic backgrounds. This is perhaps most striking when United States men from African or European ancestry are compared. African American men are known to have higher incidence, a more aggressive disease phenotype and increased mortality from $\mathrm{PCa} .{ }^{4}$

Limited data exists about the nature of $\mathrm{PCa}$ in sub-Saharan Africa. A comprehensive review of $\mathrm{PCa}$ in men of African origin suggested that it is more common in African American men, but considerably less so in sub-Saharan men. ${ }^{14}$ Reasons for this difference could be due to poor access to health care, reduced PSA screening or differences in aggressiveness of the disease phenotype in sub-Saharan men. This review concluded that problems with epidemiological data from subSaharan Africa may lead to an underestimate of the actual PCa incidence and mortality rates.

A recent study based on the South African cancer registry found a low incidence and mortality of $\mathrm{PCa}$ in black South African men. ${ }^{2}$ Age specific incidences of $\mathrm{PCa}$ in black, white, coloured, and Asian/Indian populations were: 19, 65, 46, and 19 per 100000 respectively. Age specific mortality rates were $11,7,52$, and 6 per 100000 respectively. The authors concluded that these differences may result from inadequate and variable reporting and diagnostics of $\mathrm{PCa}$ between groups in South Africa, rather than giving an accurate picture of the disease.

The black patients presenting to our service had more severe clinical and pathological features than the coloured, white and Asian patients. Despite being matched for age, black patients presented with higher PSA values, had a higher risk of being diagnosed with cancer, were more likely to have locally advanced disease, and were more likely to have high grade tumours. There are several potential explanations for these observed differences. The equal proportions of asymptomatic black and non-black patients in the series suggest that the worse disease characteristics seen in our black patients was not due to reduced screening in that population group. Because the risk of having a positive biopsy was similar amongst the black and non-black men who had been screened, the different rate of positive biopsies was accounted for by the symptomatic men. It could be that black men in Cape Town only access the healthcare system or are referred for biopsy when their symptoms are particularly severe. In a screening study at Tygerberg Hospital in Cape Town, Heyns, et al. ${ }^{15}$ found there was a low rate of prostate biopsy among the black patients with abnormal PSA values. It is therefore also possible that our black patients were less likely to be recommended for or accept the recommendation of a prostate biopsy if they were minimally symptomatic.

Despite these possible health access explanations for the racial discrepancies seen, it seems likely that there is an underlying biological factor at play. The mean ages between race groups were matched, both overall and amongst the men diagnosed with $\mathrm{CaP}$. This would suggest that black men were either developing prostate cancer at a younger age or that they were prone to a more rapidly progressive disease. The finding that the black patients in the series were at significantly greater risk of being diagnosed with high grade tumours is also an indication that the cancers diagnosed were more progressive and/or more longstanding.

Prostate sampling bias is a phenomenon that arises from the random nature of the prostate biopsy - the same volume of cancer in a larger prostate will be more easily missed at biopsy, and smaller foci of higher grade tumour will similarly be missed. Because the prostate volumes were not significantly different between black and non-black patients, the differences noted were not due to such a bias.

When comparing our data to that of developed countries, the worse clinical and pathological features seen in our black patients is seemingly on a background of generally more severe disease among all of our patients. Overall, the patients in our series presented to us largely with symptomatic disease. A study published in 2001 found that $75 \%$ of US men over the age of 50 had previously been screened for prostate cancer. ${ }^{16}$ The finding that only $21 \%$ of our patients were asymptomatic shows indirectly that the rate of PSA screening in our population is probably very low. The overall positive biopsy rate of $46.6 \%$ is somewhat higher than those of other published series of first time TRUS-guided prostate biopsies, which one review reported to be $38-42 \% .{ }^{17}$ Our high rate is probably another reflection of the low level of screening in our 
population. Our patients also presented with a higher rate of high grade disease than that seen in other settings. Only $35.1 \%$ of our patients had Gleason scores of 6 or less. A review of the CaPSURE database in the US reported that $64.9 \%$ of men on the database had Gleason scores of 6 or less. ${ }^{18}$ The higher PSA values, higher Gleason scores and higher rate of locally advanced tumours that we see in our series are indicative of delayed diagnosis. Men served by the public health system in South Africa are less likely than their European or American counterparts to be screened for prostate cancer when asymptomatic. It is also possible that when South African men do become symptomatic, they are less likely to be tested for prostate cancer - due to healthcare access problems, reduced health-seeking behaviour, or health provider practices. Black South African men could be more affected by these problems than coloured, white, or Asian patients.

We identified several limitations to our study. Firstly, it was not possible to estimate prostate cancer incidences, since the population we serve is poorly defined and there are a number of other facilities in these areas at which patients might undergo prostate biopsy. Secondly, we have no data on the subsequent management of these patients or their outcome following treatment. Thirdly, although we found significant differences between the black and the non-black patients in our series, our study was not able to determine the underlying reasons for these discrepancies. The racial, genetic, and socioeconomic makeup of South African communities is somewhat heterogeneous, and so our findings may not necessarily pertain to other areas of the country.

\section{Conclusion}

Our study has demonstrated that the men who had prostate biopsies at Groote Schuur Hospital during 2008-2014 had more severe clinical and pathological characteristics of prostate cancer, compared to American and European data.

The black patients we saw presented with significantly worse disease characteristics than their coloured, white, or Asian contemporaries. They had higher PSA values, were at higher risk of having cancer diagnosed, had higher grade tumours, and had more locally advanced disease.

The underlying reasons for these differences are speculative and are likely to be multifactorial. Delayed presentation due to healthcare access disparities and different health-seeking behaviours could account for some of those differences. The different $\mathrm{CaP}$ characteristics seen could also be due to underlying biological factors. Whether different biological risk is due to genetic or environmental factors is also uncertain. Large genetic and epidemiological studies are required to answer these questions.

\section{REFERENCES}

1. Ferlay J, Soerjomataram I, Dikshit R, Eser S, Mathers C, Rebelo $\mathrm{M}$, et al. Cancer incidence and mortality worldwide: Sources, methods and major patterns in GLOBOCAN 2012. Int J Cancer.
2015;136(5):E359-86.

2. Babb C, Urban M, Kielkowski D, Kellett P. Prostate cancer in South Africa: pathology based national cancer registry data (1986-2006) and mortality rates (1997-2009). Prostate Cancer. 2014;2014:419801.

3. Powell IJ. The precise role of ethnicity and family history on aggressive prostate cancer: a review analysis. Arch españoles Urol. 2011;64(8):711-9.

4. Powell IJ, Bock CH, Ruterbusch JJ, Sakr W. Evidence supports a faster growth rate and/or earlier transformation to clinically significant prostate cancer in black than in white American men, and influences racial progression and mortality disparity. J Urol. May 2010;183(5):1792-6.

5. Chinegwundoh F, Enver M, Lee A, Nargund V, Oliver T, BenShlomo Y. Risk and presenting features of prostate cancer amongst African-Caribbean, South Asian and European men in North-east London. BJU Int. 2006;98(6):1216-20.

6. Glover FE, Coffey DS, Douglas LL, Cadogan M, Russell $\mathrm{H}$, Tulloch $\mathrm{T}$, et al. The epidemiology of prostate cancer in Jamaica. J Urol. Jun 1998;159(6):1984-7.

7. Romero FR, Romero AW, de Almeida RMS, Filho RT. The prevalence of prostate cancer in Brazil is higher in Black men than in White men: Systematic review and meta-analysis. Int Braz J Urol. 2012;38(4):440-7.

8. Do YK, Carpenter WR, Spain P, Clark J a, Hamilton RJ, Galanko J, et al. Race, healthcare access and physician trust among prostate cancer patients. Cancer Causes Control. 2010;21(1):31-40.

9. Holz LE, Goodman M. Epidemiology of advanced prostate cancer: Overview of known and less explored disparities in prostate cancer prognosis. Curr Probl Cancer. 2015;39(1):11-6.

10. Parkin DM, Bray F, Ferlay J, Jemal A. Cancer in Africa 2012. Cancer Epidemiol Biomarkers Prev. 2014;23(6):953-66.

11. Hsing AW, Yeboah E, Biritwum R, Tettey Y, Marzo AM De, Adjei A, et al. High Prevalence of Screen Detected Prostate Cancer in West Africans: Implications for Racial Disparity of Prostate Cancer. J Urol. 2015;192(3):730-5.

12. Ukoli F, Osime U, Akereyeni F, Okunzuwa O, Kittles R, AdamsCampbell L. Prevalence of elevated serum prostate-specific antigen in rural Nigeria. Int J Urol. 1 Jun 2003 ;10(6):315-22.

13. Bradshaw E, Harington JS. A comparison of cancer mortality rates in South Africa with those in other countries. S Afr Med J. 1982;61(25):943-6.

14. Rebbeck TR, Devesa SS, Chang B-L, Bunker CH, Cheng I, Cooney $\mathrm{K}$, et al. Global patterns of prostate cancer incidence, aggressiveness, and mortality in men of African descent. Prostate Cancer. Jan 2013;2013:560857.

15. Heyns CF, Mathee S, Isaacs A, Kharwa A, De Beer PM, Pretorius M. Problems with prostate specific antigen screening for prostate cancer in the primary healthcare setting in South Africa. BJU Int. 2003;91:785-8.

16. Sirovich BE, Schwartz LM, Woloshin S. Screening Men for Prostate and Colorectal Cancer in the United States. JAMA. 19 Mar 2003;289(11):1414.

17. Shariat SF, Roehrborn CG. Using biopsy to detect prostate cancer. Rev Urol. 2008;10(4):262-80.

18. Barocas DA, Cowan JE, Smith JA, Carroll PR. What Percentage of Patients With Newly Diagnosed Carcinoma of the Prostate are Candidates for Surveillance? An Analysis of the CaPSURE Database. J Urol. 2008;180(4):1330-5. 